\title{
Morbimortalidad del embarazo gemelar en el Hospital Universitario del Valle
}

\author{
Camilo Augusto Cañas Giraldo*; Rodrigo Cifuentes B.**; Héctor Fabio Montes
}

\begin{abstract}
RESUMEN
Se presenta la experiencia con relación a morbimortalidad del embarazo gemelar en el Hospital Universitario del Valle-Cali (Colombia), donde se atendieron en un periodo de un año 7890 partos. Hubo 164 casos de embarazo múltiple (1 de cada 48 partos; o sea $2.07 \%$ ). Hubo 1 mortalidad materna por eclampsia $(0.56 \%)$ y 39 muertes fetales (11.85\% de mortalidad). El 53.8\% (21/39) de la mortalidad fetal ocurrió en menores de 750 gramos. Se presentaron trillizos en 1 ocasión (1 en 7890 partos o sea $0.012 \%)$ y siameses toracópagos en 1 ocasión. El odds ratio de nacer prematuro por ser gemelar fue de 5.48 (IC 4.9 - 6.1). La mitad de los embarazos gemelares nacieron por cesárea. A diferencia de lo reportado a nivel mundial no se encontraron diferencias en morbilidad o mortalidad en los recién nacidos con relación a su orden de nacimiento.
\end{abstract}

PALABRAS CLAVES: Morbimortalidad, embarazo gemelar, siameses.

\section{SUMMARY}

It is presented the experience in relationship to morbimortality of the multiple pregnancy in the Hospital Universitario del Valle - Cali (Colombia), where were attended in a period of one year 7890 deliveries.

There were 178 cases of multiple pregnancy ( 1 of each 44 deliveries; or $2.25 \%$ ). There was 1 maternal mortality $(0.56 \%)$ and 39 fetal deaths $(11.85 \%$ of mortality). $53.8 \%(21 / 39)$ of the fetal mortality occurred in less than of 750 grams. They were presented triplet in 1 occasion ( 1 in 7890 deliveries; or $0.012 \%$ ) and cojoined thoracopagus twins once. The relative risk of born premature by be twin gestation was of 5.48 (IC 4.9 - 6.1). Half of the multiple pregnancies were born by $C$-section. As opposed to what is reported at world level were not found differences in morbidity or mortality in the newborn in relationship their birth order.

KEY WORDS: Morbimortality, twin gestation, cojoined twins.

\section{Introducción}

La frecuencia de embarazo gemelar ha sido medida en aproximadamente 1 de cada 80 partos (1); sin embargo dan cuenta de casi $13 \%$ de toda la mortalidad perinatal en base principalmente a la prematurez (2-3) al parecer por la sobredistensión del útero (4); aunque también contribuyen la RPM (Ruptura Prematura de Membranas), RCIU (Retardo del Crecimiento Intrauterino), anomalías congénitas, problemas de cordón, abruptio de placenta, malpresentaciones y síndrome de trasfusión feto-fetal (4). Por parte de la madre; la morbilidad materna está aumentada 3-7 veces (4), siendo principalmente por desórdenes hipertensivos, sepsis asociada a RPM y excesivo sangrado postparto (4). Es por ello que el diagnóstico de embarazo gemelar le confiere a las gestantes y a sus productos unas características especiales que las

\footnotetext{
* $\quad$ Residente del Departamento de Pediatría. Universidad del Valle. ** Profesor Titular y Jefe del Departamento de Ginecología y Obstetricia. Universidad del Valle.

Profesor Titular. Departamento de Pediatría. Universidad del Valle.
}

convierten en población de alto riesgo para enfermar y morir; por ello a este grupo etáreo se deben dirigir acciones específicas que permitan reducir esas cifras de morbimortalidad.

\section{Materiales y Métodos}

Se realizó un estudio prospectivo descriptivo para conocer nuestra epidemiología respecto a la morbimortalidad del embarazo gemelar en el Hospital Universitario del Valle -Cali. (Hospital Nivel III). Para tal, se elaboró un formato en donde se consignaron durante el periodo de un año (I/15/97 a I/15/98) todos los embarazos múltiples; midiendo las siguientes variables: edad, raza, paridad, presencia de control prenatal adecuado, realización de ecografía durante el embarazo, momento del diagnóstico del embarazo gemelar, tiempo trascurrido entre el nacimiento de los bebés, tipo de placenta, historia familiar de embarazo gemelar, patología materna asociada, presentación, vía de nacimiento y cigocidad de los fetos, y complicaciones maternas. Además se determinó en cada recién nacido sexo, edad gestacional, peso y discordancia de peso, estado de salud, APGAR al 
minuto y 5 minutos; y patología y condiciones al egreso de los recién nacidos.

Se definió control prenatal adecuado la presencia de 3 o más controles antes del parto. El tipo de placenta se definió según la nota del médico que atendía el parto o de la descripción de una ecografía realizada temprano en el embarazo.

Se definió discordancia de peso así: ninguna cuando la diferencia del mayor al menor era menor del 15\%. Discordancia tipo I si la diferencia de peso era entre el 15-25\% y Discordancia tipo II si la diferencia era mayor al $25 \%$ (4). Se definió prematurez la edad gestacional menor de 37 semanas calculada por Ballard realizado en las primeras 24 horas de nacido. Se definió sangrado materno abundante aquel que requiriera trasfusión de glóbulos rojos para mejorar las cifras de hemoglobina en la madre; y atonía uterina cuando requería masaje uterino bimanual, uso de oxitócicos y trasfusión de glóbulos rojos para recuperar a la paciente. El tipo de cigocidad se determinó al analizar las variables descritas por Arias de acuerdo al sexo de los recién nacidos y tipo de placenta (4).

Los resultados se analizaron haciendo uso del paquete estadístico de Epiinfo 6.04. Se hicieron los análisis pertinentes de tendencia central y desviación standard para las variables continuas y se hicieron cruces de variables para encontrar alguna relación entre ellas.

Criterios de Selección: Se incluyeron todas las pacientes con diagnóstico de embarazo múltiple nacidos en el periodo del estudio que fue de un año. Se excluyeron 10 pacientes; así:

- 7 Partos de embarazo gemelar que llegaron remitidos pero que no fueron atendidos en el Hospital Universitario del Valle ( 4 en Hospitales periféricos y 3 atendidos en casa) por tener datos no confiables.

- 2 Partos de embarazo gemelar trasladados al servicio de pensionados luego de haber sido atendidos inicialmente en sala general.

- 1 Parto de Gemelos Siameses; que se excluyó solamente del análisis de mortalidad.

\section{Resultados}

Hubo en total 7.890 embarazos cuyo parto fue atendido en el Hospital Universitario del Valle, de ellos hubo 164 embarazos gemelares ( 1 de cada 48 embarazos, o $2.07 \%$ ); entre ellos 1 caso de trillizos y un caso de siameses Toracópagos.

El promedio de edad materna fue 24 años con rango de 12 a 45 años. La mitad de los pacientes (52\%) fue de raza mestiza, una tercera parte de raza negra $(33.5 \%)$ y $13 \%$ de raza indígena ( 21 casos) La tercera parte de las pacientes fueron primíparas $(32.3 \%)$; la de mayor paridad fue grávida 10 . Hubo control prenatal adecuado en $71 \%$ de las pacientes $(117 / 164)$, y hubo ecografía durante el embarazo en el $83 \%$ (137/164). En 30 pacientes (18.3\%) el diagnóstico se realizó solamente intraparto pues la paciente llegó en expulsivo, sin control prenatal ni ecografía. El tiempo entre nacimiento de los recién nacidos fue en promedio 12 minutos con rango de 1 minuto a 480 minutos; en más de la mitad de los casos (57\%) la diferencia de tiempo fue de máximo 5 minutos.
Cuadro 1

TIPO DE PLACENTA EN EMBARAZOS GEMELARES. HOSPITAL UNIVERSITARIO DEL VALLE. 1997-1998

\begin{tabular}{|l|c|c|}
\hline & Casos & Porcentaje \\
\hline Bicoriónica-biamniótica & 80 & 48.8 \\
\hline Bicoriónica-monoamniótica & 27 & 16.5 \\
\hline Monocoriónica-biamniótica & 23 & 14 \\
\hline Monocoriónica-monoamniótica & 11 & 6.7 \\
\hline No Especificada & 23 & 14 \\
\hline TOTAL & 164 & 100 \\
\hline
\end{tabular}

Se pudo determinar el tipo de placenta en el $86 \%$ (141/ 164) de los casos (ver cuadro número 1 ).

Hubo historia familiar positiva para embarazo gemelar sólo en el 29\% (48/164) de los casos. No hubo pacientes con inductores de ovulación. Había patología materna asociada en el 57.3\% (94/164) de los casos (ver cuadro número 2). Para la presentación y vía de nacimiento ver cuadros número 3 y 4 .

El tipo de cigocidad se pudo definir sólo en la mitad de los casos; siendo la mayoría dicigóticos y los resultados se muestran en el cuadro número 5 . Hubo complicaciones maternas asociadas en el $16 \%$ de los casos (26/ 164); siendo la principal el sangrado vaginal (Ver cuadro número 6).

De los 164 embarazos gemelares se obtuvieron en total 329 productos, $48.6 \%$ de sexo masculino (160) y $51 \%$ (168) de sexo femenino (en 1 paciente no se pudo definir sexo por inmadurez marcada de sus genitales). La edad gestacional de los recién nacidos fue en promedio 35 'semanas con rango de 20 a 40 semanas. Hubo $49.5 \%$ de recién nacidos con edad gestacional por debajo de 37 semanas (163/329). El peso de los recién nacidos fue en promedio de 2096 gr con rango de 420 a 3985 gr. Hubo en total $73 \%$ de pacientes por debajo de 2500 gr (117/ 164), 32 pacientes $(9.7 \%)$ con peso por debajo de 1000 gramos y 21 pacientes $(6.38 \%)$ menores de 750 gramos.

En los primogénitos el $68 \%$ de los recién nacidos pudo ser trasladado con su madre al servicio de recuperación, el $28 \%$ restante $(46 / 164)$ tuvo que ser hospitalizado en su periodo neonatal inmediato por alguna causa (ver cuadro número 8 ); y en el $3 \%$ de los casos (5/164) hubo óbito fetal. Respecto al segundo recién nacido pudo ser trasladado con la madre el $69 \%$ de los casos (113/ $164)$, el $29 \%$ restante se trasladó a la sala de recién nacidos por alguna causa (47/164) y hubo óbito fetal en 4 pacientes $(2.43 \%)$. Las patologías fueron semejantes a las observadas con los primogénitos. En el cuadro número 9 se pueden observar las características epidemiológicas de los grupos de gemelos. 
Cuadro 2

PATOLOGIA MATERNA EN EMBARAZOS

GEMELARES. HOSPITAL UNIVERSITARIO DEL

VALLE. 1997-1998

\begin{tabular}{|l|c|c|}
\hline & Casos & Porcentaje \\
\hline HIE & 28 & 17.1 \\
\hline RPM & 25 & 15.2 \\
\hline TPPT & 13 & 7.9 \\
\hline Hemorragia III Trimestre & 8 & 4.9 \\
\hline Diabetes Gestacional & 5 & 3.0 \\
\hline Infección Urinaria & 3 & 1.8 \\
\hline TPPT+HIE & 2 & 1.2 \\
\hline HIE+Sangrado & 2 & 1.2 \\
\hline Sd convulsivo & 2 & 1.2 \\
\hline Isoinmunización RH & 2 & 1.2 \\
\hline Sufrimiento fetal Agudo & 2 & 1.2 \\
\hline TPPT+RPM & 1 & 0.6 \\
\hline Eclampsia & 1 & 0.6 \\
\hline Ninguna & 70 & 42.7 \\
\hline TOTAL & 164 & 100 \\
\hline
\end{tabular}

HIE: Hipertensión inducida por el embarazo.

RPM: Ruptura Prematura de Membranas.

TPPT: Trabajo de Parto Pretérmino.

Hubo en total entre los primogénitos 19 mortalidades fetales $(11.58 \%)$ y entre los nacidos de segundos hubo 20 muertes (12.19\%). Hubo mortalidad simultánea en 17 gemelares, en 3 casos murió sólo el segundo gemelo y en ningún caso hubo muerte aislada del primer recién nacido. Hubo 1 mortalidad materna por eclampsia. Hubo en total $18 \%$ de recién nacidos con apgar al minuto o a los cinco minutos por debajo de 7 y sólo en $9.7 \%$ de los pacientes menor de 3 a los 5 minutos. En ningún paciente hubo diferencia de APGAR al nacer y a los cinco minutos mayor de 3 puntos entre los gemelos.

La discordancia de peso observada fue en $8.5 \%$ de los pacientes (14/164) mayor al $25 \%$ con respecto al gemelo de mayor peso, en 31 pacientes $(18.9 \%)$ entre $15-25 \%$ y en los 118 pacientes restantes (72\%) menor al $15 \%$.
Cuadro 3

PRESENTACION EMBARAZOS GEMELARES. HOSPITAL UNIVERSITARIO DEL VALLE. 1997-1998

\begin{tabular}{|l|c|c|}
\hline & Casos & Porcentaje \\
\hline Cefálica-cefálica & 76 & 46.3 \\
\hline Cefálica-podálica & 32 & 19.5 \\
\hline Podálica-podálica & 22 & 13.4 \\
\hline Podálica-cefálica & 20 & 12.2 \\
\hline Cefálica-transversa & 10 & 6.1 \\
\hline Transversa-cefálica & 1 & 0.6 \\
\hline Otras & 3 & 1.8 \\
\hline TOTAL & 164 & 100 \\
\hline
\end{tabular}

Cuadro 4

VIA DE NACIMIENTO EMBARAZOS GEMELARES. HOSPITAL UNIVERSITARIO DEL VALLE. 1997-1998

\begin{tabular}{|l|c|c|}
\hline & Casos & Porcentaje \\
\hline Partos & 87 & 53 \\
\hline Cesáreas & 72 & 43.9 \\
\hline Parto-cesárea & 5 & 3 \\
\hline TOTAL & 164 & 100 \\
\hline
\end{tabular}

Cuadro 5

CIGOCIDAD EMBARAZOS GEMELARES. HOSPITAL UNIVERSITARIO DEL VALLE. 1997-1998

\begin{tabular}{|l|c|c|}
\hline & Casos & Porcentaje \\
\hline Monocigóticos & 21 & 12.8 \\
\hline Dicogóticos & 61 & 37.2 \\
\hline No Definidos & 82 & 50 \\
\hline TOTAL & 164 & 100 \\
\hline
\end{tabular}


Cuadro 6

COMPLICACIONES MATERNAS EMBARAZO GEMELAR. HOSPITAL UNIVERSITARIO DEL VALLE. 1997-1998

\begin{tabular}{|l|c|c|}
\hline & Casos & Porcentaje \\
\hline Sangrado Abundante & 11 & 6.7 \\
\hline Atonía Uterina & 6 & 3.7 \\
\hline Retención Fetal & 5 & 3.0 \\
\hline Eclampsia & 3 & 1.8 \\
\hline Desgarro Vaginal & 2 & 1.2 \\
\hline Muerte Materna & 1 & 0.6 \\
\hline Ninguna & 138 & 84 \\
\hline TOTAL & 164 & 100 \\
\hline
\end{tabular}

Cuadro 7

MORBILIDAD EN RECIEN NACIDOS EMBARAZO GEMELAR. HOSPITAL UNIVERSITARIO DEL VALLE. 1997-1998

\begin{tabular}{|l|c|c|}
\hline & Casos & Porcentaje \\
\hline Membrana Hialina & 25 & 15.3 \\
\hline Taquipnea Transitoria & 14 & 8.6 \\
\hline Mal aptación Neonatal & 4 & 2.5 \\
\hline Sepsis & 4 & 2.5 \\
\hline Bronconeumonía & 2 & 1.2 \\
\hline Malformaciones & 1 & 0.6 \\
\hline Isoinmunización RH & 1 & 0.6 \\
\hline Asfixia Perinatal & 1 & 0.6 \\
\hline No determinada & 1 & 0.6 \\
\hline Ninguna & 110 & 67.5 \\
\hline TOTAL & & 100 \\
\hline
\end{tabular}

\section{Discusión}

En la última década este es el segundo trabajo publicado en la literatura nacional sobre la presentación epidemiológica del embarazo gemelar en nuestro medio. Estudio previo realizado por el Dr. Manotas en Medellín (5) reporta una frecuencia de embarazo gemelar de $1.16 \%$ (1 de cada 86 embarazos) que es baja si se compara con la obtenida en este trabajo; sin embargo es diferente a la reportada en diferentes latitudes (Bélgica 1:56, Italia 1:86, Grecia 1.130, Japón 1:150 y China 1:300) (6). La frecuencia de trillizos de $0.02 \%$ ( 1 en 5317 partos) en el trabajo de Medellín (5); es ligeramente mayor a la nuestra de $0.012 \%$ ( 1 en 7890).

Respecto a la edad materna se deben destacar los extremos presentados de 12 años lo que no es más que el reflejo del embarazo en adolescentes, y en pacientes de 45 años lo que deja ver la problemática de los servicios de planificación familiar.

Respecto al sexo; los gemelares más frecuentemente son mujeres respecto a hombres en una relación de $2 / 3$ a $1 / 3$, esto no se presentó en nuestro trabajo ni en el de Medellín en donde la relación fue casi a mitades. Tampoco fue más frecuente en la raza negra como descrito en otras latitudes (4).

Llama la atención que pacientes a pesar de no tener control prenatal adecuado llegaban al momento del parto con ecografía prenatal, esto pues la ecografía afortunadamente se ha popularizado aún en las clases sociales bajas. Antes del ultrasonido menos del $50 \%$ de los embarazos gemelares era diagnosticado antes de la semana 32 de gestación; y se ha visto que ayuda a disminuir la morbilidad perinatal del 5,4\% al 2.2\% según el estudio de Jensen (7). En nuestro trabajo si se compara la mortalidad entre las pacientes que tuvieron ecografía y las que no; se encuentra un odds ratio de 3.58 (IC $1.61-7.92$ ) para las pacientes sin ecografía. Se considera pues esencial el estudio ecográfico en el diagnóstico y manejo de las complicaciones perinatales del embarazo gemelar (8); ya que cuando se logra hacer control prenatal adecuado y temprano es posible definir el riesgo de prematurez al medir la longitud del canal cervical a las 24 semanas de gestación; si este es menor de $25 \mathrm{~mm}$ el riesgo relativo es casi 7 (9).

En nuestro estudio el mayor tiempo entre el nacimiento de los gemelos fue 480 minutos (por dificultades administrativas); sin embargo han sido descritos en la literatura períodos de tiempo hasta de 30 días (10).

Desde el punto de vista ecográfico y clínico pueden distinguirse 3 tipos de gemelos. Esos diferentes tipos tienen diferentes implicaciones pronósticas y se ve que la mortalidad en los bicoriónicos-biamnióticos es $9 \%$, en los momocoriónicos-monoamnióticos de 50\%; con cifra intermedia para los monocoriónicos.-diamnióticos de $26 \%$ (3). En nuestro trabajo esas cifras fueron $11.25 \%$, $0 \%$ y $8.6 \%$ respectivamente.

Se han descrito muchas patologías maternas asociadas entre ellas HTA en $37 \%$, parto pretérmino entre $20-50 \%$, retardo de crecimiento intrauterino en $47 \%$, RPM en el $22 \%$ de los pacientes, muerte fetal del $7 \%$ (11); en nuestro estudio las cifras para esas mismas patologías fueron $19.5 \%, 49.4 \%, 27 \%, 15.8 \%$ y $3 \%$ respectivamente. 
Cuadro 8

CARACTERISTICAS RECIEN NACIDOS EMBARAZO GEMELAR. HOSPITAL UNIVERSITARIO DEL VALLE. 1997-1998

\begin{tabular}{|c|c|c|c|c|}
\hline & Primer & Gemelo & Segundo & Gemelo \\
\hline Sexo Masculino & 86 & $(53 \%)$ & 73 & $(44 \%)$ \\
\hline Sexo Femenino & 77 & $(47 \%)$ & 91 & $(56 \%)$ \\
\hline Edad Gestacional & 35 & $(20-40)$ & 35 & $(20-40)$ \\
\hline Peso al nacer & 2096 & $(445-3500)$ & 2064 & $(420-3985)$ \\
\hline Hospitalizados & 46 & $(28 \%)$ & 47 & $(29 \%)$ \\
\hline Con la Madre & 113 & $(69 \%)$ & 113 & $(69 \%)$ \\
\hline Obitos & 5 & $(3 \%)$ & 4 & $(2 \%)$ \\
\hline Egreso Vivo & 144 & $(89 \%)$ & 144 & $(88 \%)$ \\
\hline Egreso Muerto & 19 & $(11 \%)$ & 20 & $(12 \%)$ \\
\hline APGAR 1 Min & 7 & $(0-9)$ & 7 & $(0-9)$ \\
\hline APGAR 5 Min & 8 & $(0-10)$ & 8 & $(0-10)$ \\
\hline
\end{tabular}

Cuadro 9

ODDS RATIO DE MORTALIDAD EN RECIEN NACIDOS DE EMBARAZO GEMELAR. HOSPITAL UNIVERSITARIO DEL VALLE. 1997-1998

\begin{tabular}{|l|c|c|c|}
\hline & Odds Ratio & IC 95 \% & P \\
\hline Presencia de Ecografía & 3.34 & $1.61-7.92$ & 0.0003 \\
\hline Prematurez & 5.48 & $4.90-6.13$ & $0.0000--$ \\
\hline Peso menor de 2500 gramos & 1.18 & $1.11-1.26$ & 0.0001 \\
\hline Peso menor de 1500 gramos & 2.13 & $1.60-2.81$ & $0.0000--$ \\
\hline Peso menor de 1000 gramos & 31.13 & $4.50-214.32$ & $0.0000--$ \\
\hline Edad materna menor de 35 años & 1.08 & $0.81-1.43$ & 0.5360 \\
\hline Edad materna menor de 15 años & 1.14 & $1.08-1.21$ & 0.5132 \\
\hline Raza Indígena & 0.83 & $0.65-1.05$ & 0.2014 \\
\hline Primigestante & 1.08 & $0.97-1.2$ & 0.2087 \\
\hline Presentación cefálica-cefálica & 0.90 & $0.80-1.01$ & 0.0741 \\
\hline Presencia de control prenatal & 1.05 & $0.91-1.21$ & 0.4411 \\
\hline Tiempo de extracción menor de 5 minutos & 1.07 & $0.95-1.21$ & 0.2346 \\
\hline Nacimiento por parto vaginal & 0.97 & $0.86-1.09$ & 0.6117 \\
\hline Sexo Masculino & 1.03 & $0.92-1.15$ & 0.6647 \\
\hline
\end{tabular}


El odds ratio para prematurez en embarazo gemelar fue de 5.48 (IC 4.9-6.1) con relación al 13\% de prematurez de embarazos únicos (12).

Otros autores han encontrado aumento de desórdenes hipertensivos no proteinúricos con mayor frecuencia pero no la preeclampsia propiamente dicha aumentada en embarazos gemelares (13). No se encontraron datos de polihidramnios; descritos del 5-8\% (3) de estas pacientes.

La presentación más frecuente en el trabajo de Medellín fue cefálica -cefálica en el $40.5 \%$ (5) de los casos semejante a la nuestra de $46.3 \%$; y a lo reportado en la literatura en $39.6 \%$ de los casos (4).

El porcentaje de gemelares nacidos por cesárea se considera entre $37-79 \%$ (14-15) que está en el rango obtenido en el presente trabajo del $47 \%$ y del $52.4 \%$ reportado por Manotas (5).

Está descrita la necesidad de parto vaginal para el primer gemelo y cesárea de emergencia para el segundo en casos de anomalías de presentación, hecho que fue visto en el $3 \%$ de nuestras pacientes; por fortuna sin implicaciones de morbimortalidad.

La frecuencia de embarazos monocigóticos a dicigóticos son en general $30 \mathrm{Vs} 70 \%$ cuando se realizan todos los estudios clasificatorios (3); en nuestro estudio esa relación se mantuvo $1 / 3$ a $2 / 3$ para los casos en los cuales se pudo definir esta; pues en la mitad de los casos no pudo definirse por falta de datos (grupo sanguíneo, ecografía temprana, etc); y aunque por definición los gemelares monoamnióticos deben nacer por cesárea (4) hay casos descritos de nacimiento por parto vaginal con ayuda ecográfica (16).

Se dice que la mitad de los gemelos pesan menos de 2.500 gramos y ello se superó en nuestro estudio en donde el $71.4 \%$ de los recién nacidos fueron menores de ese peso. Dada la frecuencia de retardo de crecimiento intrauterino para embarazos gemelares; que aumentan a medida que aumenta el número de productos (17); se están elaborando cartas especiales de crecimiento en 19.000 gemelos (18) para poder definir con exactitud su patrón de crecimiento en la gestación.

Respecto a la discordancia de peso; cuya causa puede ser desiguales masas placentarias, síndromes genéticos y síndrome de trasfusión fetofetal (4); se ha visto que tiene mayores implicaciones de morbimortalidad cuando es mayor del $25 \%$ la diferencia de peso en base al gemelo mayor (14). En nuestro estudio la discordancia de más de $25 \%$ fue de $8.5 \%$ (14/164) y en ese grupo de pacientes la morbilidad fue de $42.8 \%(12 / 28)$ y la mortalidad de $21.4 \%(6 / 28)$.

Las anomalías descritas con mayor frecuencia son: cardíacas, del tubo neural, urogenital, trastornos cromosómicas, defectos del paladar, etc (4). En nuestra casuística fue $0.6 \%$ ( 1 caso de polidactilia); cifra baja si se compara con la encontrada por el Dr. Manotas del $4 \%$
(5), que es aún baja al compararla con lo reportado en la literatura mundial de $17 \%$ (19).

La mortalidad en los recién nacidos de gemelos del 4.7\% obtenida en el trabajo de Medellín (5) es baja con relación a la nuestra en donde se obtuvo una mortalidad de $11.8 \%$; sin embargo si se excluyen prematuros extremos (menos de $750 \mathrm{gr}$ ) y óbitos la mortalidad se reduce al $2.7 \%$ de los gemelares. Si se filtran un poco los datos de mortalidad el estudio del Dr. Manotas (5) solo tenía partos por encima de 28 semanas de edad gestacional; y eso puede explicar en parte las diferencias encontradas; aunque en la literatura internacional se reportan cifras de mortalidad perinatal de gemelos es $14.2 \%$ (4), más acorde con nuestros hallazgos. El odds ratio de morir en menores de $1500 \mathrm{gr}$ fue 2.13 y de 31.1 para menores de 1000 gramos. Otros Odds ratio pueden observarse en el cuadro número 9; en donde llama la atención que la presencia de control prenatal per se no afecta los resultados de mortalidad para los recién nacidos. Lo mismo para la raza, la gravidez, la edad materna, el tiempo de extracción fetal, la presentación y la vía de nacimiento; cuyos odds ratio son todos cercanos a 1 con intervalos de confianza estrechos.

La morbilidad $(28 \%)$ y mortalidad perinatal del primer recién nacido $(11.5 \%)$ fue semejante a la del segundo ( $29 \%$ y $12.1 \%$ respectivamente); y es alta y contradictoria al ser comparada con los datos de $3.3 \%$ y $6.6 \%$ respectivamente descritos por Briese (20); y del $9 \%$ y $14 \%$ descritos por Arias (4) en donde el segundo gemelo sufre más morbimortalidad. Esas diferencias no se explican por las características epidemiológicas de cada grupo, de recién nacidos como se mostró en el cuadro número 8.

Algunos autores han encontrado influencia de edad materna y raza a la hora de definir riesgos en gemelares (22) sin embargo en nuestro trabajo en el análisis multivariado no se encontró ninguna diferencia de mortalidad con relación a gravidez, presencia de control prenatal, raza o edad materna, presentación, vía de nacimiento, sexo o tiempo de extracción fetal.

La morbilidad general del $25.3 \%$ en Medellín y la nuestra de $28.5 \%$ son similares; aunque en Medellín la principal morbilidad fetal fue la asfixia perinatal, en nosotros fue la Enfermedad de Membrana Hialina.

El óbito descrito en el 0.5 al $6.8 \%(4,21)$ de los gemelares es comparable con nuestra cifra de $2.73 \%$ (9/ 329). Cuando se analizan los efectos a largo plazo del handicap de los productos de embarazo gemelar, que pueden ser de 3.7 a 4.4 para gemelos, 8.7 para trillizos y 11.1 para cuatrillizos (23); se describe que la variable con mayor peso es la edad gestacional, más que el sexo, la paridad o el orden de nacimiento; al menos en menores de $1.500 \mathrm{gr}$ al nacer que en nuestro estudio fueron el $18.23 \%$ de los partos (60 pacientes) (24). 


\section{REFERENCIAS}

1. Rojas J. Embarazo Gemelar En: Stark, C., Montes, H., Fundación

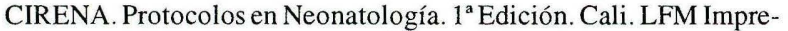
siones; 1996; 31-33.

2. Skupski D, Chervena KF. Multiple Gestation Pregnancies in: Spitzer, A. Intensive Care of the Fethus and Neonate. Edition. 1996; 315-25.

3. Keith R, Haidet K, Alfred K. Routine Ultrasound Evaluation of the Uncomplicated Pregnancy in: Spitzer, A. Intensive Care of the Fethus and Neonate. Edition 1996; 38-62.

4. Arias F. Practical Guide to High Risk Pregnancy and Delivery. $2^{\text {a }}$ Edition 1993; 131-49.

5. Manotas R, Hernández E. Situación de Morbilidad en Gemelos Recién Nacidos. IATREIA 1991; 4: 61-69.

6. Behrman R, Kliegman R. Nelson Textbook of Pediatrics. $14^{\circ}$ Edition. W.B. Saunders; $1992 ; 439-41$.

7. Jensen OH. Twin Pregnancy: Results from the Periods 1987-88 and 1900-93. Tidsskr Nor Laegeforen 1995; 115: 2061-63.

8. Divon MY, Weiner Z. Ultrasound in Twin Pregnacy. Semin Perinatol 1995; 19: 404-12.

9. Golderberg RL, Iants JD, Miodovnik M. et al. The Preterm Prediction Study: Risk factors in Twin Gestations. National Institute of Child Health and Human Development Maternal-Fetal Medicine Units Network. Am J Obstet Gynecol 1996; 175:1047-53.

10. Kisoka R. Separate Birth 30 days after a Premature Delivery in a Twin Pregnancy. A Case Report. J Gynecol Obstet Biol Reprod. 1994; 23: 719-20.

11. Ariza A. Embarazo Múltiple en: Cifuentes R. Obstetricia de Alto Riesgo. $4^{a}$ edición. 1994; 379-402.

12. Departamento de Estadística del Departamento de Ginecología y Obstetricia del Hospital Universitario del Valle.

13. Dsantema JG, Koppelaar I, Wallenburg HC. Hypertensive Disorders in Twin Pregnancy. Eur J Obstet Gynecol Reprod Biol 1995; 58: 9-13.

14. Cheung VY, Bocking AD, Dasilva OP. Preterm Discordant Twins: What Birth Weight Difference is Significant? Am J Obstet Gynecol 1995; 172: 955-59.

15. Divon MY, Marin MJ, Pollack RN. et al. Twin Gestation: Fetal Presentation as a Function of Gestational Age. Am J Obstet Gynecol $1993 ; 168: 1500-02$.

16. Dubecq F, Dufour P, Vinatier D. et al. Monoamniotic Twin Pregnancies. Review of the Literature, and a Case Report with Vaginal Delivery. Eur J Obstet Gynecol Reprod Biol 1996; 66:183-86.

17. Sherer DM, Divon MY. Fetal Growth in Multifetal Gestation. Clin Obstet Gynecol. 1997; 40:764-70.

18. Buckler JM, Green M. Birth Weight and Heah Circunference Standards for English Twins. Arch Dis Child 1994; 71: 516-21

19. Yokayama Y, Shimizu T, Hayakawa K. Handicaps in Twins and Triplets. Nippon Eiseigaku Zasshi 1995; 49: 1013-18.

20. Briese B, Falkert U, Piese R, Muller H. Analysis of 122 Deliveries with Special Reference to Morbility and Mortality of the Second Twin. Zentraibi Gynokol 1994; 116: 38-43.

21. So BH, Tamura M, Kamoshita S. Liveborn Twin with Intrauterine Death of one Twin: Report of Two Cases. Chung Mua Min Kuo Hsiao Erh I Hsueh Tsa Chih 1994; 35: 312-18.

22. Powers WF, Wamplert NS. Further Defining the Risk Confronting Twins. Am J Obstet Gynecol 1996;175: 1522-28.

23. Yokahoma Y, Shimizu T, Hayakawa K. Incidence of Handicaps in Multiple Births and Associated Factors. Acta Genet Med Gemellol 1995; 44: 81-91.

24. Chen SJ, Vohr VR, OH W. Effects of Birth Order, Gender and Intrauterine Growth Retardation on the Outcome of Very Low Birth Weigth in Twins. J Pediatr 1993; 123:132-36.

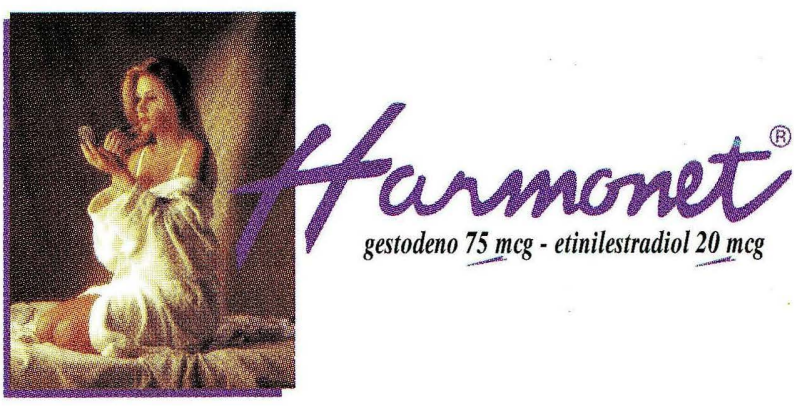

FORMULA

Cada gragea contiene: Gestodeno $75 \mathrm{mcg}$, Etinilestradiol $20 \mathrm{mcg}$, Lactosa 37,155 mg, azúcar 19,66 mg almidón de maiz $15,5 \mathrm{mg}$, carbonato de calcio $8,697 \mathrm{mg}$, talco $4,242 \mathrm{mg}$ polietilenglicol $60002,18 \mathrm{mg}$, povidona K-25 1,7 $\mathrm{mg}$, estearato de magnesio $550 \mathrm{mcg}$, povidona K-90 $171 \mathrm{mcg}$, cera E $50 \mathrm{mcg}$.

INDICACIONES

Harmonet está indicado para la prevención del embarazo en mujeres que deciden utilizar este método.

POSOLOGIA Y FORMA DE ADMINISTRACION

Para lograr una eficacia anticonceptiva optima, HARMONET deberá ingerirse diariamente según las indicaciones y con intervalos diarios que no excedan las 24 horas. Debe instruirse a la paciente para que tome las grageas todos los días a la misma hora, preferiblemente con la comida de la noche o bien antes de acostarse.

\section{CONTRAINDICACIONES}

Harmonet está contraindicado en las mujeres que presenten:

Antedentes o diagnóstico de trastornos trombóticos arteriales o venosos, trastornos embólicos o situaciones que pudieran predisponer a la paciente (por ejemplo, defectos de la coagulación, valvulopatias cardiacas, fibrilacion auricular); transtornos agudos o crónicos de la función hepática (incluyendose entre estos el sindrome de DubinJohnson 0 al sindrome de Rotor), antecedentes o diagnostico del tumores hepaticos antecedentes de ictericia idiopática o de prurito importante durante el embarazo; antecedentes o diagnostico conocido o sospechado de neoplasias hormonodependientes ( por ejemplo, cancer de mama o de endometrio); trastornos del metabolismo lipídico; anemia falciforme; diabetes melitus con alteraciones vasculares; antecedentes de herpes gestaciona; otosclerosis que se agravan durante el embarazo; sangrado vaginal no diagnósticado; embarazo conocido o sospechado; hipersensibilidad a cualquiera de los componentes de Harmonet.

EFECTOS SECUNDARIOS

Los siguientes efectos secundarios se han registrado en un total de 1338 pacientes que recibieron Harmonet durante el curso de tres estudios clínicos:

Generales Incidencia $>3 \%$ : dolor abdominal, dolor de espalda, dolor en los genitales, dolor pélvico. Incidencia < que 1\%. distension del abdomen, abcesos, reacciones alergicas, astenia, fiebre, sindrome gripa pesadez en las extremidades, infecciones, malestar, moniliasis, artrits reumatoidea. Sistema Cardiovascular Incidencia $>3 \%$ : migraña. Incidencia de 1\%-3\%: venas varicosas. Incidencia <1\%: dolor al pecho trombosis de las venas profundas solocos, hipentension, palpitaciones, taquicardia, tromboembolia, trombollebitis, vasodilatación, y otros transtornos cardiovasculares. Sistema Digestivo Incidencia <3\%: Nauseas. Incidencia 1\%-3\%: Vomito. ncidencia < $1 \%$ : colecistis, diarrea, flatulencia, transtornos vesiculares,gastritis, gastroenteritis, transtornos gastrointestinales, hepatopatia aumento del apetito estomatitis. Sistema Metabolico Incidencia < $1 \%$ edema aumento o pérdida del peso. Sistema Nervioso Incidencia $>3 \%$ : Cefaleas, nerviosismo. Incidencia 1\%-3\%: Depresión, mareos, alteraciones en la libido. Incidencia < $1 \%$ : amnesia, ataxia, hostilidad, parestesia, transtornos en el sueño, somnolencia, sudoración excesiva. Sistema Respiratorio incidencia < < \%: bronquitis, faringitis, rinitis, sinusitis. Dermatologicos. Incidencia >3\%: Acne. Indicidencia < <\%: alopecia, cloasma, eczema, prurito, eritema otros trastornos dermatológicos. Organos de los sentidos. Incidencia < 1\%: vision anormal, tinnitus, sordera tota transitoria. Sistema Urogenital. Incidencia $>3 \%$ : amenorrea, sangrando intermestrual, goteo, tension mamaria. Incidencia < $1 \%$ : cistitis, dismenorrea, disuria, dolor en los genitales, galactorrea, leucorrea, nefritis, quistes ováricos, cálculos renales infecciones del trato urinario, sequedad vaginal, moniliasis vaginal, vaginitis, transtornos vulvovaginales. Por otra parte, los siguientes efectos secundarios se han registrado en pacientes que recibian anticonceptivos orales y se consideraron relacionados con la droga administrada:Náuseas, Vómitos, sintomas gastrointestinales, (Tales como cólicos abdominales e inflamación), sangrado intermenstrual, goteo, alteraciones en el flujo menstrual, amenorrea infertilidad temporaria con posterioridad a la descontinuación del tratamiento, edema melasma que puede persistir, aumento o disminución de peso, alteraciones en la erosión y secreción cervical, disminución en el volumen de leche en el postparto inmediato, ictericia colestática, migraña, eritema (alérgico), depresión mental, menor tolerancia a los carbohidratos, candidiasis vaginal, acentuación de la curvatura de la córnea intolerancia a los lentes de contacto, cambio en las mamas: tensión, aumento de tamaño, secreción. Existen evidencias de una posible relación entre los siguientes efectos secundarios y la utilización de anticonceptivos orales, aunque aún debe confirmarse esta información: Trombosis mesentérica, Trombosis retiniana. Los siguientes efectos secundarios se han registrado en pacientes que recibian anticonceptivos orales, pero su asociación no ha sido aún confirmada ni rechazada: Anomalias congénitas. Síndrome premestrual, cataratas, asociación no ha sido aun contio neuritis optica, cambios en el apetito, sindrome similar a la cistitis, cetaleas, neviosismo, mareos, hirsutismo, perdida síndrome urémico hemolítico, sindrome de BuddChiari, acné, cambios en la líbido. colitis, enfermedad cerebrovascula con prolapso de la válvula mitral, sindrome similares a lupus.

Para mayor información, véanse las advertencias y precauciones.

SOBREDOSIS

La sobredosis puede causar náuseas o vómitos; en las mujeres puede producirse sangrado por supresión. En niños, no se han registrado efectos graves después de la utilización de altas dosis de anticonceptivos orales.

PRESENTACION

Envase con 21 grageas. Registro INVIMA 006606 